Preprint: final version available as:

Diriye, A., Blandford, A., Tombros, A., \& Vakkari, P. (2013). The Role of Search

Interface Features during Information Seeking. In Research and Advanced

Technology for Digital Libraries (pp. 235-240). Springer Berlin Heidelberg.

Publisher URL:

http://link.springer.com/chapter/10.1007/978-3-642-40501-3_23

DOI: $10.1007 / 978-3-642-40501-3 \_23$ 


\title{
The Role of Search Interface Features During Information Seeking
}

\author{
Abdigani Diriye ${ }^{1}$, Ann Blandford ${ }^{2}$, Anastasios Tombros ${ }^{3}$ and Pertti Vakkari ${ }^{4}$ \\ ${ }^{1}$ Carnegie Mellon University, Pennsylvania, USA \\ ${ }^{2}$ University College London Interaction Centre, University College London, UK \\ ${ }^{3}$ Department of Computer Science, Queen Mary University London, UK \\ ${ }^{4}$ School of Information Sciences, University of Tampere, Finland \\ adiriye@cs.cmu.edu, a.blandford@ucl.ac.uk, \\ tassos@eecs.qmul.ac.uk, ti.vakkari@uta.fi
}

\begin{abstract}
In this paper, we examine the role search interface features play in information seeking across different categories and complexities of search tasks. We present a system called Search Buddy that provides features to enable exploration, filtering and browsing of information. Differing categories and complexities of search tasks were studied through qualitative and quantitative methods. We find specific user patterns in the frequency, points and context of search interface usage. This study highlight the potential value of contextualizing interface features to the type of task and stage of information seeking.
\end{abstract}

\section{Introduction}

A variety of interface features exist to help search engine and digital library users search, browse and find useful information. Examples include related searches, document summaries and query autocomplete. Each of these are intended for different purposes and play different roles in the user's information seeking.

System designers and researchers have several design approaches in their toolbox to help them construct search engine and digital library interfaces: for example the eight "golden rules" for interface design; the user-centered design process; task analysis; and interface design patterns like Yahoo's and Endeca's. Given these tools, we find that there is little theoretical work that puts forward a solid explanation to how different search engine and digital library interface features are used or what their role or importance is. To design and construct supportive search interfaces, we need to understand more about the utility of these interface features. In this paper we present a user study that examines the role of interface features on peoples information seeking.

\section{Background}

Search interfaces and search tasks are well studied topics. Search tasks vary in terms of complexity, clarity, and the search activities involved. A number of studies show these factors invariably affect how searchers interact with information [4] [6]. Studies into different search interfaces have found features on search 
engine and digital library interfaces like faceted navigation are useful for browsing tasks [9]; query autocomplete have been shown to be perceived as useful for well-defined tasks [8]; and related searches are known to be useful for complex tasks [3]. Research has also looked at the relationship between search tasks and search interfaces. Washington carried out a field study that looked at how search interface features support jobs tasks at a large public institution [7], and found that depending on the task at hand, users want additional content or different layouts. Divoli and Medelyan conducted a qualitative study evaluating the usefulness of digital library interface features by bioscientists [2], and came to the conclusion that there are preferences for certain search features over others. Paul and Morris developed a collaborative search system that supports sensemaking via several activity views [5], and found their participants used different search views for different collaboration modes and task types. Vakkari et al. analyzed how information searching and IR is connected with tasks [6]. They found that different task stages affect peoples search tactics, term selections and relevance judgements. More recently, Diriye et al. in [1] carried out work that explored the relationship between search tasks and search interfaces and found that interfaces have an impact on information seeking.

Some work has been carried out on search interfaces and search tasks, but there still is a dearth of work that closely examines their relationship. This paper extends previous work by: (i) examining the role and utility of search interface features; and (ii) studying several different search interface features.

\section{System Design}

We constructed a rich and highly interactive search interface that enables all the major search activities involved in known-item and complex and exploratory search tasks such as lookup, browsing and learning [4]. Search Buddy provides a search box and list of search results that provide the user with the ability to lookup and navigate documents; search filters and related searches on the lefthand pane to assist in browsing, filtering, identification and exploration; and "starter pages", a novel feature that promotes learning by presenting documents that are of a general nature. The search interface features designed support different kinds of search actions and activities. In Figure 1, we see a screenshot of the search interface. Search Buddy works by retrieving search results using the Bing API. The search filters are generated by using the search result captions (i.e. the title, snippet and URL) to generate labels for topics latent in the search results. The Lingo clustering algorithm was used to organize search results into topics. The related searches, found on the left-hand side, are scraped from Google, and the starter pages were created by identifying authoritative and popular resources.

\section{Study Design}

The study had a $2 \times 2$ factorial design: two different categories of search tasks (known-item and exploratory) and two different levels of complexity (low and 


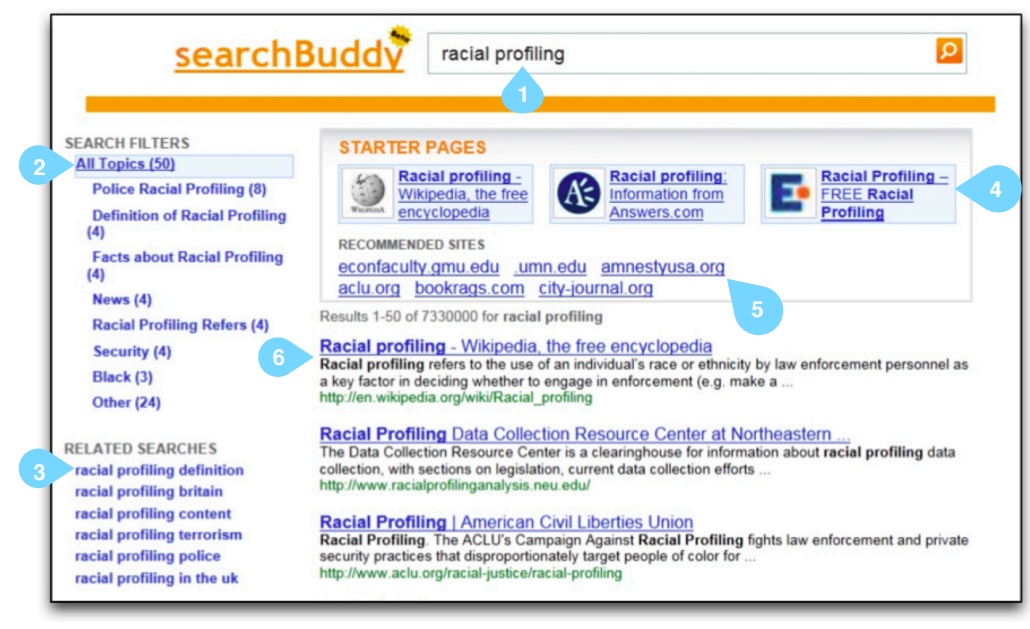

Fig. 1: Screenshot of the Search Buddy interface. $1=$ search box; $2=$ search filters; $3=$ related searches; $4=$ starter pages; $5=$ recommended sites; $6=$ search result caption.

high). We developed a rich search interface to provide a diverse range of search actions and activities during usage. We decided to focus on only one search interface as our interest lay in studying how the search interface was used during information seeking. We collected qualitative and quantitative data using questionnaires, interviews, eye-tracking and system logs, and we logged our participants interactions at the search feature-level and the task-level.

\subsection{Methodology}

Each user study lasted up to an hour, and our participants were paid $£ 7$ each for their time. A Graeco Latin square design was used to permute the search tasks. The study procedure began with the participant being informed of the aim of the study, afterwards, each participant was asked to select a search tasks from each of the four categories of search tasks and rate them, and explain how they plan on completing the task. They would then perform the task whilst using the Tobii eye-tracking machine; gather information to address the task into a text file; complete a questionnaire on how useful and helpful the search features were; and finally provide feedback on the rationale behind their usage of the search features. After the study, each participant was required to fill out an exit questionnaire comparing the search features for the different search tasks. Finally, the participant was thanked and paid for their time.

\subsection{Participants}

We recruited 24 participants via a university participant pool. The participants ages ranged from $18-51$ (mean $=27.1$ years) and our sample comprised 8 males and 16 females. The participants all expressed familiarity with popular search 
engines like Google, Yahoo and Bing, and reported having at least 6 years of online searching experience (mean $=8.8$ years), and they all rated their computer proficiency between average and excellent.

\section{$5 \quad$ Results}

The data is focused around two core themes: 1) the impact the search tasks have on information seeking; and 2) the usage and impact of the search features during the search tasks.

\subsection{Perceptions of Search Features}

After each search task, our participants were asked to complete a questionnaire eliciting their perceptions on how useful, helpful and important the search features were during the search task. Statistical analysis revealed that there were significant differences between the search features (all: $F_{3,22}=16.432, \mathrm{p}<.000$ ), but our participants could not perceive any differences between the search tasks (all: $F_{3,22}<1.480, \mathrm{p}<.158$ ). This means despite the fact that some search features were perceived as more or less useful for certain search tasks, this trend was not apparent for all search tasks.

Search Interface Feature Usage. According to the data from our system logs, that specific search feature usage was not statistically significant across any of the search tasks (starter pages: $F_{3,23}=1.319, \mathrm{p}=.275$; related searches: $F_{3,23}=1.668, \mathrm{p}=.182$; search filters: $\left.F_{3,23}=1.470, \mathrm{p}=.238\right)$. But, there were significant differences in the total usage of search interface features for each search task (total: $F_{3,23}=4.334, \mathrm{p}=.049$ ). What this means is that though we could not find a relationship between specific search features and specific search tasks, there was an increase in the number of search support features used as the search task became more complex and exploratory. We also found a significant difference between the number of queries and documents selected across the different search task (queries: $F_{3,23}=8.029, \mathrm{p}<.001$; docs selected: $\left.F_{3,23}=12.604, \mathrm{p}<.001\right)$.

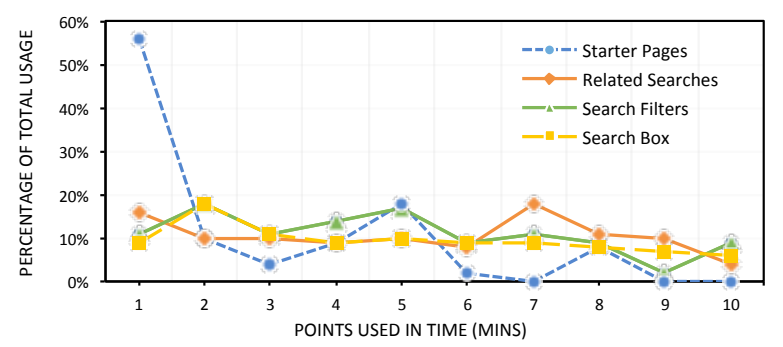

Fig. 2: Time intervals search features were used across all the search tasks. 


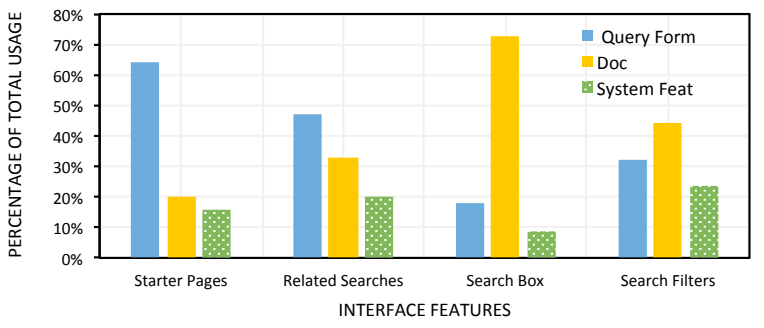

Fig. 3: Preceding user actions prior to use of interface features.

Points of Search Interface Usage. Using our system logs and screen recording, we examined the points in time search features were used.We can also see in the figure a peak in the usage for the search box. $50 \%$ of the queries formulated via the search box was concentrated in the $2^{\text {nd }}-4^{\text {th }}$ minutes of our participants search tasks, whereas usage of the search filter and related searches remained steady across the entire duration of the search tasks. A chi-squared test showed these figures were significant $\left(\chi_{2}=113.6, \mathrm{p}<.017\right)$. This data highlights the differences in how these system features were used amongst our participants across the search tasks. The starter pages were found to be mostly used at the start of the search tasks to find general information, and subsequently used less frequently when more specific information was required. Other features like the search filters and related searches were utilized throughout the search task.

Context of Usage. Our system logs and screen recording reveal search features were used after leaving a document, manually formulating a query, or after using other search features. In Figure 3, we present the frequency of usage. The starter pages and search box were associated with specific contexts. Queries were reformulated using the search box feature after our participants would leave a document over $73 \%$ of the time. The most popular sequence of search behaviours involved returning from a document and then reformulating the query using the search box feature. Similar search sequences were evident for the starter pages. $64 \%$ of usage of the starter pages occurred after the searcher had formulated a query. In all, search features like the search box and starter pages can be context and search stage specific, whereas features like the search filters and related searches were used uniformly across the different search stages and contexts.

\section{Discussion}

The findings in this paper verify search features are context and search stage specific: search interface features like the search box and starter pages were used heavily at the start of the search tasks, and within specific contexts like after a query reformulation or document selection. Other search features like the search filters and related searches were more flexible and used uniformly across the different search stages and contexts. This indicates that search features play 
different roles on the user interface. Some search features like the starter pages and search box are search stage-specific features that are useful at certain points during information seeking or search contexts; other search features like the search facets and search filters are search stage agnostic features, and afford the user a generic set of search actions and activities at any stage of their information seeking.

Although we did not find significant differences in how useful, helpful or important the search features were perceived to be across the different search tasks, we did find significant differences in how frequently the search features were used. The data reveals that as the search tasks became more complex and exploratory, and required more search action and strategies to complete, the total number of search features used on the features increased.

In the post-task interviews our participants identified using the search features based on the attributes of the search task they were undertaking, or as a result of their search habits, and in some cases as a fallback mechanism when the search box and search results failed to help them find relevant information. This further substantiates the finding that search features support as well as impede information seeking [1].

Our findings have implications for the design of search interfaces: interface features have been shown to be search stage and search task sensitive, this puts the case forward for adaptive interfaces that present interface features that suit a user's particular search context and stage. This could be search contexts where the system deciphered the user is engaged in like look-up or identification, and thus promotes the use of certain interface features like search filters, or cases

where the users search tasks appears to be exploratory or informational, and so search features like starter pages or related searches are promoted.

\section{References}

[1] A. Diriye, A. Blandford, and A. Tombros. When is System Support Effective? In Information Interaction in Context, IIiX 2010.

[2] A. Divoli, and A. Medelyan, Search interface feature evalua-tion in biosciences, HCIR 2011, Google, Mountain View, CA, USA. 2011.

[3] H. Fowkes and M. Beaulieu. Interactive searching behavior: Okapi experiment for TREC-8, Proc. BCS-IRSG, pp. 47 56. 2000

[4] G. Marchionini. Exploratory search: from finding to understanding. Commun. ACM, vol. 49, no. 4, pages 41 46, 2006

[5] S. A. Paul and M. R. Morris. 2009. CoSense: enhancing sensemaking for collaborative web search. In Proc. of CHI. pgs 1771-1780. 2009.

[6] P. Vakkari, M. Pennanen and S. Serola. Changes of search terms and tactics while writing a research proposal. Inf. Process. Manage., pages 445 - 463, 2003.

[7] A. Washington, Discussing Design: How search interface features support job tasks in an institutional repository. Proc. of AMCIS. 2006.

[8] R. W. White, M. Bilenko and S. Cucerzan. Studying the use of popular destinations to enhance web search interaction. In Proc. of SIGIR 07, pages 159 166, 2007.

[9] K-P. Yee, K. Swearingen, K., Li, and M. Hearst, Faceted Metadata for Image Search and Browsing, in CHI 2003. 